Documentation et bibliothèques

DOCUMENTATION BIBLIOTHÈQUES

\title{
À l'aube de transformations majeures des bibliothèques du Québec : quand les architectes et les bibliothécaires sont complices
}

\section{Guylaine Beaudry et Yvon-André Lacroix}

Volume 60, numéro 2-3, avril-septembre 2014

Bibliothèques et architecture

URI : https://id.erudit.org/iderudit/1025509ar

DOI : https://doi.org/10.7202/1025509ar

Aller au sommaire du numéro

\section{Éditeur(s)}

Association pour l'avancement des sciences et des techniques de la documentation (ASTED)

\section{ISSN}

0315-2340 (imprimé)

2291-8949 (numérique)

Découvrir la revue

\section{Citer ce document}

Beaudry, G. \& Lacroix, Y.-A. (2014). À l'aube de transformations majeures des bibliothèques du Québec : quand les architectes et les bibliothécaires sont complices. Documentation et bibliothèques, 60(2-3), 60-61. https://doi.org/10.7202/1025509ar

Tous droits réservés ( $)$ Association pour l'avancement des sciences et des techniques de la documentation (ASTED), 2014
Ce document est protégé par la loi sur le droit d'auteur. L'utilisation des services d'Érudit (y compris la reproduction) est assujettie à sa politique d'utilisation que vous pouvez consulter en ligne.

https://apropos.erudit.org/fr/usagers/politique-dutilisation/ 


\title{
À l'aube de transformations majeures des bibliothèques du Québec : quand les architectes et les bibliothécaires sont complices
}

\author{
GUYLAINE BEAUDRY \\ Directrice et bibliothécaire en chef \\ Bibliothèques, Université Concordia \\ guylaine.beaudry@concordia.ca

\section{YVON-ANDRÉ LACROIX} \\ Consultant en bibliothéconomie et rédacteur \\ Directeur général de la diffusion de Bibliothèque \\ et Archives nationales du Québec (BAnQ) de 1999 à 2003 \\ yalacroix@gmail.com
}

$\mathrm{D}$ EPUIS LE DÉBUT DU MILLÉNAIRE, LE QUÉBEC VOIT de plus en plus de réalisations remarquables de nouvelles bibliothèques ou de rénovations majeures pour créer des lieux documentaires d'un calibre architectural significatif. Grâce au Congrès des milieux documentaires 2011 (voir encadré) et à l'instauration du prix Architecture de bibliothèques et de centres d'archives du Québec, les milieux québécois de la bibliothéconomie et de l'architecture connaissent une convergente complicité qui encourage un climat extrêmement favorable à la créativité. Nous tenons à remercier Michèle Hudon, directrice de Documentation et bibliothèques de 2008 à 2013, qui nous a lancé le défi d'explorer cette thématique de l'architecture des bibliothèques au Québec.

Ce numéro thématique et le récent livre de Jacques Plante témoignent avec éclat de cette présence d'avantgarde qu'occupent dorénavant ces " architectures de la connaissance »dans le paysage du bâti au Québec ${ }^{1}$. À quelques mois près, nos collègues de l'enssib (École nationale supérieure des sciences de l'information et des bibliothèques) lançaient un ouvrage collectif, Architecture et bibliothèque, 20 ans de constructions ${ }^{2}$. Les bibliothèques changent, évoluent et deviennent des lieux en complète redéfinition, que ce soit sur le plan des collections, des services ou des espaces.

Quel que soit le mot utilisé - transformation, mutation ou métamorphose -, les bibliothécaires redéfinissent, avec les architectes, ces lieux publics afin de trouver des solutions en vue d'intégrer les technologies et les collections numériques et d'en mesurer l'impact sur les services et les équipements à rendre disponibles aux usagers. Les bibliothèques de tous les milieux sont plus que jamais des lieux où s'expriment et se vivent de puissants liens sociaux, et elles facilitent le développe-

1 et 2. Voir les comptes rendus présentés à la fin de ce numéro. ment des communautés et des individus. L'architecture et le design jouent un rôle de première importance pour créer des bibliothèques bien adaptées aux changements constants à venir et toujours aptes à la stimulation culturelle et à l'inspiration intellectuelle.

Ce numéro thématique propose d'explorer les questions à considérer pour l'élaboration de programmes d'espace qui correspondent aux besoins des usagers de façon innovante et de plain-pied dans la culture numérique. L'approche multisectorielle s'impose pour la réussite de tels projets. Ainsi, les contributions à ce numéro présentent différentes perspectives, notamment bibliothéconomique, architecturale, de design ou de gestion. Un chassé-croisé où les chercheurs et les praticiens témoignent de leurs réflexions et de leurs expériences dans des projets, petits, moyens ou grands, planifiés ou réalisés au cours des dernières années. Les projets sont issus des milieux collégiaux, publics et universitaires, et concernent tout autant un bâtiment neuf, un agrandissement ou une rénovation qu'un recyclage, une conservation ou une restauration patrimoniale. Le présent numéro est une occasion de rompre une certaine timidité des bibliothécaires en rapport à l'architecture et de créer des ponts pour une implication beaucoup plus substantielle de leur part dans les étapes du processus architectural.

Le modèle architectural unique pour les bibliothèques, abandonné depuis quelques années, a laissé place à une saine émulation entre les bibliothécaires et les architectes pour innover et répondre adéquatement aux besoins actuels des collectivités à desservir, et permettre un ancrage encore plus solide des bibliothèques au Québec. De tout temps, ces deux partenaires sont constamment présents et proactifs dans la transformation et l'adaptation de cette institution qui, plus qu'un espace physique, est le lieu d'une expérience humaine, toujours et encore unique. 


\section{Le Congrès des milieux documentaires 2011}

Le thème retenu pour le Congrès des milieux documentaires 2011, " redéfinir l'espace documentaire ", portait essentiellement sur l'architecture des lieux et de leurs services, aussi bien en milieu scolaire que collégial, universitaire que municipal, bibliothèque de dépôt que Maison de la littérature. Une profusion d'ateliers destinés à réfléchir à la construction, l'agrandissement, l'aménagement, à aborder les nouvelles tendances sociales, les technologies, le développement durable, à jongler avec l'alchimie des espaces et à mettre en perspective le dialogue nécessaire des expertises propres aux bibliothécaires et aux architectes.

Outre de nombreux bibliothécaires, près d'une dizaine d'architectes, de designers d'intérieur et d'ergonomes, tels Manon Asselin, Jacques Bélanger, Anne Carrier, Chevalier/Morales/Luc Plamondon, Vincent Leclerc, Éric Pelletier, Monica Ponce de
Leon, ont expliqué leur démarche et beaucoup témoigné de leur engagement à travailler en équipe avec les professionnels des bibliothèques et à bien comprendre les besoins des clients bibliothécaires.

Le bibliothécaire est, selon eux, un client ouvert, généreux, créateur, un partenaire indispensable puisque le programme d'une bibliothèque, pôle identitaire d'une communauté, est d'une extrême complexité. Une démarche d'étroite collaboration est essentielle puisque chaque projet, unique, s'adresse à des clientèles diverses et souvent de tous les âges, et qu'il demande du bibliothécaire et de l'architecte de porter autant d'attention au plus grand qu'au plus petit détail et que la notion de bien-être est difficile à cerner et toujours en évolution. Un tel lieu exige un dialogue des compétences pour que les éléments fonctionnels s'harmonisent naturellement avec l'esthétique et la beauté.

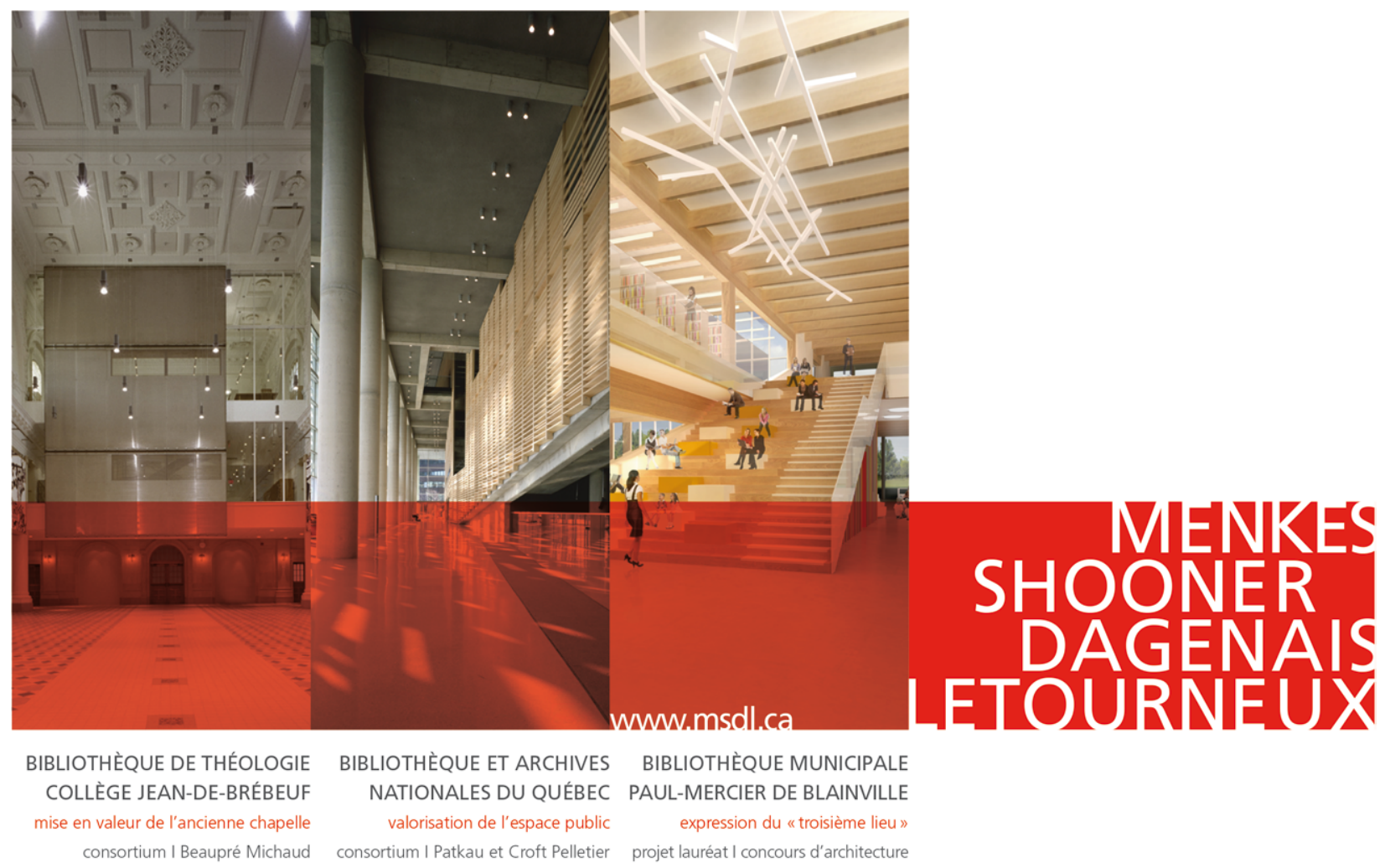

